Journal of Mathematics and Informatics

Vol. 17, 2019, 113-129

ISSN: 2349-0632 (P), 2349-0640 (online)

Published 31 October 2019

Journal of

www.researchmathsci.org

DOI: http://dx.doi.org/10.22457/jmi.151av17a11

Mathematics and

Informatics

\title{
Modelling the Transmission Dynamics of Banana Xanthomonas Wilt Disease with Contaminated Soil
}

\author{
John Joel Mapinda, ${ }^{1, *}$, Gasper Godson Mwanga ${ }^{2}$ and Verdiana Grace Masanja ${ }^{1}$ \\ ${ }^{1}$ The Nelson Mandela Institution of Science and Technology \\ P.O. Box 447, Arusha, Tanzania \\ ${ }^{2}$ University of Dar es Salaam, Dar es salaam University College of Education \\ P.O. Box 2329, Dar es Salaam, Tanzania \\ *Corresponding author, E-mail: jmapinda@gmail.com \\ Received 18 July 2019; accepted 12 August 2019
}

\begin{abstract}
Banana Xanthomonas Wilt disease (BXW) is a bacterial disease which highly threaten banana production in east and central Africa. It is caused by a bacteria known as Xanthomonas campestris pv. musacearum $(\mathrm{Xcm})$. Mathematical modelling gives an insight on how to best understand the transmission dynamics and control of the disease. The existing mathematical models have not included contaminated soil in the dynamics of BXW. In this study we formulated a model which includes contaminated soil, calculated the basic reproduction number and carried out sensitivity analysis of some model parameters. We further conducted numerical simulation to validate the results. The simulations show that the infection rate by contaminated farming tools $\left(\beta_{i}\right.$ and $\beta_{e}$ ), the infection rate by contaminated soil $\left(\omega_{2}\right)$, vertical disease transmission rate $(\theta)$, and the shedding rate of $\mathrm{Xcm}$ bacteria in the soil $(\phi)$ are positively sensitive to the basic reproduction number. While, the most negative sensitive parameters are the clearance rate of Xcm bacteria from the soil $\left(\mu_{h}\right)$, removal of infected plants from the farm $(r)$, harvesting $\left(\alpha_{p}\right)$, and banana plants disease induced death rate $(d)$.The result also shows that contaminated soil contributes to the transmission and persistence of BXW disease. Therefore, we recommend that, along with the existing control measures scientist and technologist should carry out studies to find a way to reduce or avoid vertical disease transmission and increase the $\mathrm{Xcm}$ clearance rate in the soil. Furthermore, technology for early detection of infected plants should be brought down to the local farmers at affordable costs. This will help stakeholders to detect and remove the infected plants from the farm in time and hence reduce the number of secondary infections.
\end{abstract}

Keywords: Contaminated Soil; BXW; Mathematical Modelling; Banana Production; basic re-production number; sensitivity analysis

AMS Mathematics Subject Classification (2010): 97R20 
John Joel Mapinda, Gasper Godson Mwanga and Verdiana Grace Masanja

\section{Introduction}

Bananas are among the most important food crops in the world after maize, cassava and sweet potatoes. Farmers use Banana fruits as food and for commercial purposes to support their livelihoods. Also, to a lesser extent, bananas are used to make fibre, wine and beer. In 2016 the world produced 113,280,302 tonnes (113,280kt) of bananas where $21,019,246$ tonnes $(21,019 \mathrm{kt})$ were produced in Africa [1]. Despite the importance of this plant, the Banana Xanthomonas Wilt (BXW) disease is still a threat to banana production in Africa. The BXW disease can cause up to $100 \%$ loss of banana produce if not timely controlled. Even though the social and economic impact of BXW is not well quantified, it highly affects the food security status, livelihood of banana growing households and national economies at large [2]. BXW is caused by the bacterium known as Xanthomonas campestris pv. musacearum $(\mathrm{Xcm})$. It is transmitted through contaminated farming tools, insect vectors, soil, Infection from mother plant to its suckers, planting and transportation of latently (Asymptomatic) infected banana plants [3-5] . A symptomatic infected plant is identified by the yellowing and wilting of its leaves, premature ripening of the fruits and yellow ooze observed when pseudo stem is cut [6], [7]. The commonly used method to control the disease is through timely removing of the male bud using a forked stick, sterilization of the farming tools, debudding and rouging.

To best control the disease there is a need to understand its transmission dynamics. Mathematical modelling plays an important role in the study of the dynamics, predicting, assessing, and suggesting the best control measures to potential disease outbreaks. Researchers such as[7]-[11] have developed mathematical models to study the dynamics of BXW disease. These mathematical models did not take into account the role of contaminated soil in the dynamics of BXW disease. Nevertheless, the findings by [3, 12, 13] clearly shows that, contaminated soil plays a role in BXW transmission and persistence. [14] showed that there is a positive correlation between rain and BXW disease transmission. The rain increases the soil moisture and hence favorsthe survival of $\mathrm{Xcm}$ bacteria in the soil. Furthermore, through the flow of rain water, $\mathrm{Xcm}$ bacteria can be transported from one place to another. Therefore, in order to best understand the dynamics of BXW disease, there is a need to develop a mathematical model which takes consideration the role of contaminated soil. In this paper we developed a deterministic mathematical model which considers BXW transmission through contaminated soil, insects, farming tools and vertical transmission.

This paper is organized as follows. In Section 2, parameters and variables are described, assumptions satisfied by the parameters and variables are made, and model is formulated. In section 3, we calculated the basic reproduction number $\left(R_{0}\right)$, checked the stability of the disease free equilibrium point, carried out sensitivity analysis and did simulations. Finally section 4 includes the conclusion and recommendations.

\section{Material and methods}

\subsection{Model formulation}

The model formulated in this study involves banana plant population, insect vector population and contaminated soil. Depending on the infection status, the banana plant population is subdivided into three compartments namely: Susceptible plant $\left(S_{p}\right)$; asymptomatic infected plants $\left(E_{p}\right)$; and Symptomatic infected plants $\left(I_{p}\right)$. Susceptible are healthy banana plants which can be infected by BXW when come into contact with 
Modelling the Transmission Dynamics of Banana Xanthomonas Wilt Disease with Contaminated Soil

the Xcm bacterium. It is assumed that susceptible banana plants have an equal chance of being infected when come into contact with Xcm bacterium. Susceptible banana plants acquire BXW disease through vertical transmission, contaminated farming tools, soil contaminated with Xcm bacteria, or insect vector $[3,4,5]$.

The vectors such as birds, bats and other flying insects such as bees are the carrying agent of the $\mathrm{Xcm}$ bacteria from an infected banana plant to the susceptible plant. Birds transmit the $\mathrm{Xcm}$ bacteria after feeding on ripe banana bunches of an infected banana plant to the male buds of a susceptible plant [5]. Bats can transmit the disease through feeding on nectar or ripe banana fruits of an infected banana plant to the healthy banana plant [5]. Other vectors such as bees transmit the disease to a susceptible banana plant when contaminated with Xcm bacteria from a male bud of an infected plant [15]. In this paper, the vector population is subdivided into susceptible vectors $\left(S_{v}\right)$ and contaminated vectors $\left(I_{v}\right)$. An environment contaminated with Xcm bacteria is denoted by $A_{h}$.

The model considers constant recruitment of banana plants by emerging of new healthy lateral shoots from the banana plants and replanting of healthy suckers at the rate of $b_{p}$. Through farming activities such as weeding, pruning, removing access suckers, harvesting and male bud removal, a farming tool can be contaminated with $\mathrm{Xcm}$ bacteria from symptomatic infected banana plants or asymptomatic infected banana plants and transmit the disease at the rate $\beta_{i}$ or $\beta_{e}$ respectively. We further assume that susceptible banana plant can be infected by the Xcm bacteria found in the soil at a rate of $\omega_{2}$. The average daily contact rate of an infected vector to a susceptible banana plant is given by $a$ and the probability that the contact results to infection is given by $\omega_{1}$.

Matured banana plants are harvested at the rate of $\alpha_{p} . E_{p}$ proceed to $I_{p}$ at the rate of $q$ after showing BXW disease symptoms. An infected banana plant can be removed from the farm at the rate of $r$ right after showing symptoms or die due to infection at a rate of $d$. It is assumed that the banana plant can be vertically an infected from infected banana plant at a rate q. We further assumed that the rate of vertical transmission is less than the sum of disease induced death rate and removal of infected banana plants

$(\theta \leq d+r)$.

A susceptible vector population has a constant recruitment rate $b_{v}$, and it is assumed that both susceptible and contaminated vectors die naturally at the rate $\mu_{v}$. $\omega_{3}$ is the probability that a susceptible vector gets contaminated with $\mathrm{Xcm}$ bacteria upon coming into contact with a symptomatic infected banana plant. It is assumed that the vector becomes infective right after being contaminated with the $\mathrm{Xcm}$ bacteria. According to [5], contaminated vectors retain Xcm bacteria viable for 3-5 days from the day of inoculation. This implies that, after 5 days contaminated vectors become susceptible again at a rate $\eta$ where $\eta<\mu_{v}$. 
John Joel Mapinda, Gasper Godson Mwanga and Verdiana Grace Masanja

The shedding rate of $\mathrm{Xcm}$ bacteria from the infected banana plant in the soil is given by $\phi$. The Xcm bacteria in the farm soil are cleared naturally at the rate $\mu_{h}$ due to lack of saprophytic or resting stage in soil [16].

The model is best described by the compartmental diagram in Figure 1, where $\lambda_{1}=a \omega_{3} \frac{I_{p}}{N_{p}}$ and $\lambda_{2}=\left(a \omega_{1} \frac{I_{v}}{N_{p}}+\beta_{e} \frac{S_{p} E_{p}}{N_{p}}+\beta_{i} \frac{S_{p} I_{p}}{N_{p}}+\omega_{2} \frac{A_{h}}{N_{p}\left(K+A_{h}\right)}\right)$.

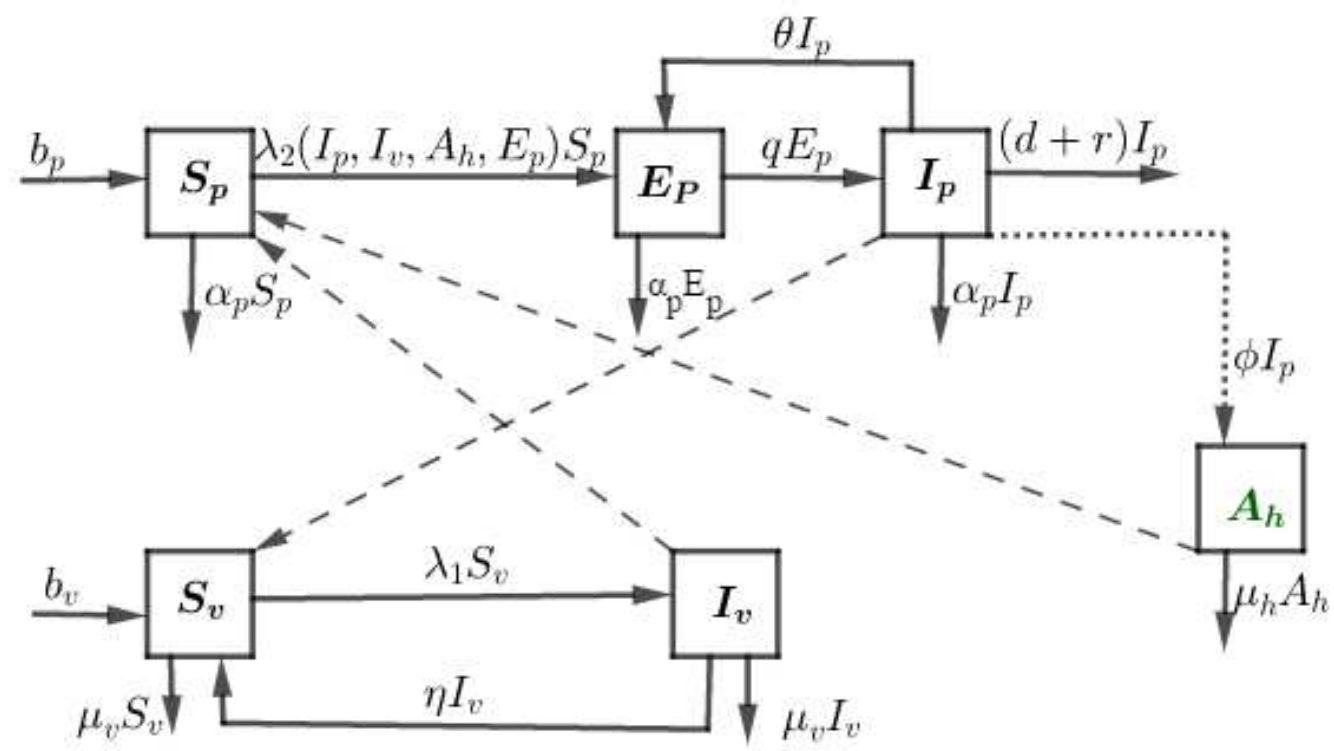

Figure 1: Basic Compartmental diagram for the transmission dynamics of BXW disease.

From the compartmental diagram, sold lines represent a transition from one infection stage to recruitment, harvesting, natural death rate of vectors and clearance of $\mathrm{Xcm}$ bacteria from the soil. The dash lines represent normal interactions between different compartments and shedding of $\mathrm{Xcm}$ bacteria onto the environment is represented by dotted lines.

From the compartmental diagram we formulate a system of differential equations as follows:

$$
\begin{aligned}
& \frac{d S_{p}}{d t}=b_{p}-a \omega_{1} \frac{S_{p} I_{v}}{N_{p}}-\beta_{e} \frac{S_{p} E_{p}}{N_{p}}-\beta_{i} \frac{S_{p} I_{p}}{N_{p}}-\omega_{2} \frac{S_{p} A_{h}}{N_{p}\left(K+A_{h}\right)}-\alpha_{p} S_{p} \\
& \frac{d E_{p}}{d t}=a \omega_{1} \frac{S_{p} I_{v}}{N_{p}}+\beta_{e} \frac{S_{p} E_{p}}{N_{p}}+\beta_{i} \frac{S_{p} I_{p}}{N_{p}}+\omega_{2} \frac{S_{p} A_{h}}{N_{p}\left(K+A_{h}\right)}+\theta I_{p}-\alpha_{p} E_{p}-q E_{p} \\
& \frac{d I_{p}}{d t}=q E_{p}-\alpha_{p} I_{p}-d I_{p}-r I_{p}
\end{aligned}
$$


Modelling the Transmission Dynamics of Banana Xanthomonas Wilt Disease with Contaminated Soil

$$
\begin{aligned}
\frac{d A_{h}}{d t} & =\phi I_{p}-\mu_{h} A_{h} \\
\frac{d S_{v}}{d t} & =b_{v}+\eta I_{v}-a \omega_{3} \frac{S_{v} I_{p}}{N_{p}}-\mu_{v} S_{v} \\
\frac{d I_{v}}{d t} & =a \omega_{3} \frac{S_{v} I_{p}}{N_{p}}-\eta I_{v}-\mu_{v} I_{v}
\end{aligned}
$$

The equations of the total population of banana plants and total population of vectors are given by:

$$
\begin{aligned}
& \frac{d N_{p}}{d t}=b_{p}-\alpha_{p} N_{p}+\theta I_{p}-(d+r) I_{p} \\
& \frac{d N_{v}}{d t}=b_{v}-\mu_{v} N_{v}
\end{aligned}
$$

\subsection{Basic properties of the model}

In this section we are going to check whether the model system is epidemiologically and mathematically well posed. This is done by checking the invariant region of the model and positivity of the model solution to make sure that there is no negative solution to the model variables. From equation (7) we have

$$
\begin{aligned}
& \frac{d N_{p}}{d t} \leq b_{p}-\alpha_{p} N_{p} \\
& N_{p}(t) \leq \frac{b_{p}}{\alpha_{p}}+\left(N_{p}(0)-\frac{b_{p}}{\alpha_{p}}\right) e^{-\alpha_{p} t}
\end{aligned}
$$

From equation(10), two cases are emerging. Case 1: When $N_{p}(0) \leq \frac{b_{p}}{\alpha_{p}}$ as $t \rightarrow \infty$ the total number of banana plants $N_{p}(t)$ increases to $\frac{b_{p}}{\alpha_{p}}$. This implies that

$$
N_{p}(0) \leq N_{p}(t) \leq \frac{b_{p}}{\alpha_{p}}, \forall_{t \geq 0}
$$

Case 2: When $N_{p}(0) \geq \frac{b_{p}}{\alpha_{p}}, N_{p}(t)$ decreases to $\frac{b_{p}}{\alpha_{p}}$ as $t \rightarrow \infty$. This implies that

$$
N_{p}(t) \leq \frac{b_{p}}{\alpha_{p}} \leq N_{p}(0), \forall_{t \geq 0}
$$

Generally, $D_{1}=\left\{S_{p}(t), E_{p}(t), I_{p}(t) \in \mathbb{R}_{+}^{3}: N_{p}(0) \leq N_{p}(t) \leq \frac{b_{p}}{\alpha_{p}}, \forall_{t \geq 0}\right\} . \quad$ Again, $\quad$ from equation (8) we have

$$
\frac{d N_{v}}{d t} \leq b_{v}-\mu_{v} N_{v}
$$


John Joel Mapinda, Gasper Godson Mwanga and Verdiana Grace Masanja

Solving this, results into

$$
N_{v}(t) \leq \frac{b_{v}}{\mu_{p}}+\left(N_{v}(0)-\frac{b_{v}}{\mu_{v}}\right) e^{-\mu_{v} t} .
$$

From equation (14) it follows that, when $N_{v}(0) \geq \frac{b_{v}}{\mu_{v}}$, as $t \rightarrow \infty$, the total number of vectors $N_{v}(t)$ reduces to $\frac{b_{v}}{\mu_{v}}$. This means that

$$
N_{v}(t) \leq \frac{b_{v}}{\mu_{v}} \leq N_{v}(0), \forall_{t \geq 0} .
$$

Again, when $N_{p}(0) \leq \frac{b_{p}}{\alpha_{p}}$, as $t \rightarrow \infty$ the number of vectors $N_{v}(t)$ approaches to $\frac{b_{v}}{\mu_{v}}$.

This means that

$$
N_{v}(0) \leq N_{v}(t) \leq \frac{b_{v}}{\mu_{v}}, \forall_{t \geq 0}
$$

Therefore,

$$
D_{2}=\left\{S_{v}(t), I_{v}(t) \in \mathbb{R}_{+}^{2}: N_{v}(0) \leq N_{v}(t) \leq \frac{b_{v}}{\mu_{v}}, \forall_{t \geq 0}\right\}
$$

Furthermore, it is proved that

$$
D_{3}=\left\{A_{h}(t) \in \mathbb{R}_{+}^{1}, \forall_{t \geq 0}\right\}
$$

Considering the non-negative initial solutions of the model $S_{p}(0)>0, E_{p}(0) \geq 0, I_{p}(0) \geq 0$, $A_{h}(0) \geq 0, S_{v}(0) \geq 0, I_{v}(0) \geq 0$, the model system (1) - (6)is positive invariant and attracting in the region

$$
D=\left\{D_{1} \times D_{2} \times D_{3}: D \in \mathbb{R}_{+}^{6}, \forall_{t \geq 0}\right\}
$$

Therefore, the model solutions remain positive and bounded in the region $\mathrm{D}, \forall_{t \geq 0}$

\section{Results and discussion}

\subsection{Disease free equilibrium point}

A disease free equilibrium (DFE) is the point at which there is no infection in the population. Thus, the populations comprise of only susceptible banana plants and vectors and no Xcm bacteria in the soil. Let, $X_{d f e}=\left(S_{p}^{0}, E_{p}^{0}, I_{p}^{0}, S_{v}^{0}, I_{v}^{0}, A_{h}^{0}\right)$ be the disease free equilibrium point of the system (1) - (6). Setting the rate of change of each model variable in the system in (1) - (6) to zero and solving the system results into DFE in (20)

$$
X_{d f e}=\left(S_{p}^{0}, E_{p}^{0}, I_{p}^{0}, S_{v}^{0}, I_{v}^{0}, A_{h}^{0}\right)=\left(\frac{b_{p}}{\alpha_{p}}, 0,0, \frac{b_{v}}{\mu_{v}}, 0,0\right)
$$

\subsection{Basic reproduction number $\mathbf{R}_{0}$}

A basic reproduction number $\left(R_{0}\right)$, is an average number of new infection caused by one infective individual in a population where all its members are susceptible. It helps to understand the ability of the disease to invade the population [17]. In this 
Modelling the Transmission Dynamics of Banana Xanthomonas Wilt Disease with Contaminated Soil

paper we apply the next generation method as described by $[17,18]$. From the system of equations(1) - (6), consider the infected sub-system

$$
\begin{aligned}
\frac{d E_{p}}{d t} & =a \omega_{1} \frac{S_{p} I_{v}}{N_{p}}+\beta_{e} \frac{S_{p} E_{p}}{N_{p}}+\beta_{i} \frac{S_{p} I_{p}}{N_{p}}+\omega_{2} \frac{S_{p} A_{h}}{N_{p}\left(K+A_{h}\right)}+\theta I_{p}-\alpha_{p} E_{p}-q E_{p} \\
\frac{d I_{v}}{d t} & =a \omega_{3} \frac{S_{v} I_{p}}{N_{p}}-\eta I_{v}-\mu_{v} I_{v} \\
\frac{d I_{p}}{d t} & =q E_{p}-\alpha_{p} I_{p}-d I_{p}-r I_{p} \\
\frac{d A_{h}}{d t} & =\phi I_{p}-\mu_{h} A_{h}
\end{aligned}
$$

Let $x=\left(E_{p}, I_{v}, I_{p}, A_{h}\right)$ and $y=\left(S_{p}, S_{v}\right)$, where $x$ and $y$ are infected and susceptible compartments of the model, respectively. Separating the infected subsystem (21) - (24) into two parts, results into (25) and (26), where $\mathcal{F}(x, y)$ is the transmission part which portray the generation of new infections and $\mathcal{V}(x, y)$ is the transition part which involves change of states.

$$
\begin{gathered}
\mathcal{F}(x, y)=\left(\begin{array}{c}
a \omega_{1} \frac{S_{p} I_{v}}{N_{p}}+\beta_{e} \frac{S_{p} E_{p}}{N_{p}}+\beta_{i} \frac{S_{p} I_{p}}{N_{p}}+\omega_{2} \frac{S_{p} A_{h}}{N_{p}\left(K+A_{h}\right)}+\theta I_{p} \\
a \omega_{3} \frac{S_{v} I_{p}}{N_{p}} \\
0 \\
\mathcal{V}(x, y)=\left(\begin{array}{c}
-\alpha_{p} E_{p}-q E_{p} \\
-\eta I_{p}-\mu_{v} I_{v} \\
q E_{p}-\alpha_{p} I_{p}-d I_{p}-r I_{p} \\
\phi I_{p}-\mu_{h} A_{h}
\end{array}\right)
\end{array}\right)
\end{gathered}
$$

Let $F=\frac{\partial \mathcal{F}(x, y)}{\partial x_{i}}$ and $V=\frac{\partial \mathcal{V}(x, y)}{\partial x_{i}}$ where $x_{i}=\left(E_{p}, I_{v}, I_{p}, A_{h}\right)$ for $i=1,2,3,4$ At the DFE every member of the population is susceptible, thus $S_{p}^{0}=N_{p}(0)$. Differentiating and evaluating at $X_{d f e}$ results into

$$
F=\left(\begin{array}{cccc}
\beta_{e} & a \omega_{1} & \beta_{i}+\theta & \frac{\omega_{2}}{K} \\
0 & 0 & \frac{a \omega_{3} \alpha_{p} b_{v}}{\mu_{v} b_{p}} & 0 \\
0 & 0 & 0 & 0 \\
0 & 0 & 0 & 0
\end{array}\right)
$$


John Joel Mapinda, Gasper Godson Mwanga and Verdiana Grace Masanja

$$
\begin{aligned}
V & =\left(\begin{array}{cccc}
\left(\alpha_{p}+q\right) & 0 & 0 & 0 \\
0 & \left(\eta+\mu_{v}\right) & 0 & 0 \\
-q & 0 & \left(\alpha_{p}+d+r\right) & 0 \\
0 & 0 & -\phi & \mu_{h}
\end{array}\right) \\
V^{-1} & =\left(\begin{array}{cccc}
\frac{1}{\left(\alpha_{p}+q\right)} & 0 & 0 & 0 \\
0 & \frac{1}{\left(\eta+\mu_{v}\right)} & 0 & 0 \\
\frac{q}{\left(\alpha_{p}+q\right)\left(\alpha_{p}+d+r\right)} & 0 & \frac{1}{\left(\alpha_{p}+d+r\right)} & 0 \\
\frac{q \phi}{\left(\alpha_{p}+q\right)\left(\alpha_{p}+d+r\right) \mu_{h}} & 0 & \frac{\phi}{\left(\alpha_{p}+d+r\right) \mu_{h}} & \frac{1}{\mu_{h}}
\end{array}\right)
\end{aligned}
$$

Then it follows that

$$
F V^{-1}=\left(\begin{array}{cccc}
T_{R} & \frac{a \omega_{1}}{\eta+\mu_{v}} & T_{C} & \frac{\omega_{2}}{k \mu_{h}} \\
\frac{a \omega_{3} \alpha_{p} b_{v} q}{\mu_{v} b_{p}\left(\alpha_{p}+q\right)\left(\alpha_{p}+d+r\right)} & 0 & \frac{a \omega_{3} \alpha_{p} b_{v}}{\mu_{v} b_{p}\left(\alpha_{p}+d+r\right)} & 0 \\
0 & 0 & 0 & 0 \\
0 & 0 & 0 & 0
\end{array}\right)
$$

Now, let the matrix $Q=F V^{-1}$, the basic reproduction number $R_{0}$ of the model is a dominant eigenvalue of the matrix $\mathrm{Q}$. Therefore,

$$
R_{0}=\frac{1}{2} T_{R}+\frac{1}{2} \sqrt{\left(T_{R}\right)^{2}+4 \frac{a^{2} \omega_{1} \omega_{3} \alpha b_{v} q}{\left(\eta+\mu_{v}\right) \mu_{v} b_{p}(\alpha+q)(\alpha+d+r)}}
$$

where

$$
T_{R}=\frac{\beta_{e}}{\alpha_{p}+q}+\frac{\left(\beta_{i}+\theta\right) q}{\left(\alpha_{p}+q\right)\left(\alpha_{p}+d+r\right)}+\frac{\omega_{2} \phi q}{k\left(\alpha_{p}+d+r\right)\left(\alpha_{p}+q\right) \mu_{h}}
$$

And

$$
T_{C}=\frac{\beta_{i}+\theta}{\alpha_{p}+d+r}+\frac{\omega_{2} \phi}{k\left(\alpha_{p}+d+r\right) \mu_{h}}
$$

From(31), $\frac{1}{\alpha_{p}+q}$ is the average time that a banana plant stays in an asymptomatic infected stage before proceeding to the symptomatic infected stage. In this duration $\beta_{e}$ 
Modelling the Transmission Dynamics of Banana Xanthomonas Wilt Disease with Contaminated Soil

new infection are generated. $\frac{q}{\alpha_{p}+q}$ is the probability that an asymptomatic infected banana plant proceeds to an infected compartment. $\frac{1}{\alpha_{p}+d+r}$ is the duration by which an infected banana plant stays in an infected group during its lifetime. $\beta_{i}+\theta$ is the expected number of new infections to the banana plant produced by a symptomatic infected banana plant before being harvested, removed from the farm or dying due to BXW disease infection. $\frac{\omega_{2} \phi}{k \mu_{h}}$ are the expected new infections caused by the contaminated soil.

\subsection{Local stability of the DFE point}

Theorem 3.1. If $X_{d f e}$ is a DFE of the model given by(1)-(6), then $X_{d f e}$ is locally asymptotically stable if $R_{0}<1$, and unstable if $R_{0}>1$

Proof: The proof of Theorem 3.1 is similar that of Theorem 2.1 of [17]

\subsection{Global stability of the DFE point}

Global stability of the disease-free equilibrium point means that the solutions of the system are attracted to the DFE point over indefinite time.

Theorem 3.2. If $X_{d f e}$ is a DFE of the model given by (1) - (6), then $X_{d f e}$ is locally asymptotically stable if $R_{0}<1$, and unstable if $R_{0}>1$.

Proof: Lyapunov function constructed using matrix theoretic method based on the Perron eigenvector is applied to prove the Global Stability of the DFE $X_{d f e}$ as done in $[20,21]$. Now, let $x=\left(E_{p}, I_{v}, I_{p}, A_{h}\right)$ and $y=\left(S_{p}, S_{v}\right)$. From a subsystem(21) - (24), the function $f(x, y)$ and $x$ can be written as in (33)and (34)respectively.

$$
f(x, y)=(F-V) x-\mathcal{F}(x, y)+\mathcal{V}(x, y)
$$

and

$$
x^{\prime}=(F-V) x-f(x, y)
$$

Solving for $f(x, y)$ results to (35)

$$
f(x, y)=\left(\begin{array}{c}
\frac{\beta_{e}\left(S_{p}^{*}-S_{p}\right) E_{p}}{N_{p}}+\frac{a \omega_{1}\left(S_{p}^{*}-S_{p}\right) I_{p}}{N_{p}}+\frac{\beta_{i}\left(S_{p}^{*}-S_{p}\right) I_{p}}{N_{p}}+\frac{\omega_{2}\left(S_{p}^{*}-S_{p}\right) A_{h}}{N_{p}} \\
\frac{a \omega_{3}\left(S_{p}^{*}-S_{p}\right) I_{p}}{\mu_{v} N_{p}} \\
0 \\
0
\end{array}\right)
$$


John Joel Mapinda, Gasper Godson Mwanga and Verdiana Grace Masanja

Referring to Theorem 2.1 of [21]. Since from (27) $F \geq 0$, in (29) $V^{-1} \geq 0$ and from (35) $f(x, y) \geq 0$ then (36) is a Lyapunov function of the model (21)-(24) where $\vartheta^{T}$ is the left eigenvector of the matrix $V^{-1} F$ Corresponding to its spectral radius $R_{0}$.

$$
Q=\vartheta^{T} V^{-1} x
$$

Reducing the matrix $V^{-1} F$ to its row echelon form, the left eigenvector is $\vartheta^{T}=(1,0,0,0)$.

$$
Q=\frac{E_{p}}{\alpha_{p}+q}
$$

Since $\vartheta^{T}>0$ and the matrix $V^{-1} F$ is irreducible and non negative, then Theorem 2.2 of [21] can be applied. Differentiating (36) results to (38)

$$
Q^{\prime}=\vartheta^{T} V^{-1} x^{\prime}
$$

Substituting (34) into (38) gives (39)

$$
\begin{aligned}
& Q^{\prime}=\vartheta^{T} V^{-1}((F-V) x-f(x, y)) \\
& =\vartheta^{T} V^{-1}(F-V) x-\vartheta^{T} V^{-1} f(x, y) \\
& =\left(R_{0}-1\right) \vartheta^{T} x-\vartheta^{T} V^{-1} f(x, y)
\end{aligned}
$$

Substituting the required equations in (41) yields (42).

$$
Q^{\prime}=\left(R_{0}-1\right) E_{p}-\frac{1}{\alpha_{p}+q}\left(\frac{\beta_{e}\left(S_{p}^{0}-S_{p}\right) E_{p}}{N_{p}}+\frac{a \omega\left(S_{p}^{0}-S_{p}\right) I_{p}}{N_{p}}+\frac{\beta_{i}\left(S_{p}^{0}-S_{p}\right) I_{p}}{N_{p}}+\frac{\omega_{2}\left(S_{p}^{0}-S_{p}\right) A_{h}}{N_{p}}\right)
$$

From (42) it can be observed that $Q^{\prime} \leq 0$ if $R_{0} \leq 1$. But if $R_{0}=1, Q^{\prime}=0 \Leftrightarrow S_{p}^{0}=S_{p}$ or $E_{p}=I_{p}=I_{v}=A_{h}=0$. Thus, by Theorem 2.2 of [20], $X_{d f e}$ is Global Asymptotically Stable in D when $R_{0} \leq 1$ and unstable when $R_{0}>1$.

\subsection{Parameter values}

The parameter values are obtained from related literature and some are assumed in the interval $(0,1)$. The values for the parameters $d, \alpha_{p}, r, \mu_{v}$ and $b_{v}$, are adopted from [10]. $\beta_{i}, \omega_{1}, \omega_{3}$ and $a$ are from [11]. $b_{p}$ from [8] and $\theta$ from [15]. Table 1 shows the parameter value per day. 
Modelling the Transmission Dynamics of Banana Xanthomonas Wilt Disease with Contaminated Soil

Table 1: Values of the model parameters

\begin{tabular}{cccccc}
\hline Parameter & Value/Range & Parameter & Value/Range & Parameter & Value/Range \\
\hline$d$ & 0.0167 & $\omega_{2}$ & $0: 4$ & $b_{v}$ & 0.02 \\
$\phi$ & 0.89 & $\beta_{i}$ & 0.1429 & $\omega_{1}$ & $0: 2$ \\
$\alpha_{p}$ & 0.0056 & $K$ & 1000 & $\mu_{v}$ & 0.02 \\
$\theta$ & 0.0286 & $\mu_{h}$ & 0.01 & $\omega_{3}$ & 0.2 \\
$r$ & 0.0105 & $q$ & 0.3 & $\eta$ & 0.0286 \\
$b_{p}$ & 0.01667 & $a$ & 0.2 & $\beta_{e}$ & 0.3 \\
\hline
\end{tabular}

\subsection{Sensitivity analysis}

Sensitivity analysis is the process of determining the influence of each model parameter in the basic reproduction number $\left(R_{0}\right)$. This guides the selection of the disease control measures, where the most sensitive parameters are highly considered. This study applied the Normalized forward sensitivity index to determine the sensitivity of the model parameters as in[21]. If the $R_{0}$ is differentiable with respect to its parameter $\mathrm{u}$, then the sensitivity index of $u$ is given by(43).

$$
\Upsilon_{u}^{R_{0}}=\frac{\partial R_{0}}{\partial u} \times \frac{u}{R_{0}}
$$

Since the $R_{0}$ in (31)is differentiable to all its parameters, now we apply (43)to calculate the sensitivity indices of the model parameters using the values in table 2 . This results to sensitivity indices as indicated in Figure 2.

A positive index implies the direct proportionality of the basic reproduction number with the corresponding parameter. A negative index means that the parameter is inversely proportional to the basic reproduction number. Increasing the $R_{0}$ implies increase in the BXW disease endemicity while decreasing $R_{0}$ to less than one lowers the endemicity of the BXW disease. From Figure 2, the parameters $\omega_{2}, \phi, \theta, \beta_{i}$ and $\beta_{e}$ have positive indices, which sends the message that increasing (or decreasing) any of these parameters keeping other parameters constant, results into the increase (or decrease) of the basic reproduction number $\left(\mathrm{R}_{0}\right)$. For instance, $\beta_{i}=0.6024153$ means that increasing (or decreasing) the value of the parameter $\beta_{i}$ increases (or decreases) the $\mathrm{R}_{0}$ by $6.024153 \%$. Thus, decreasing the rate of infection by farming tools, vertical transmission, rate of shedding $X \mathrm{~cm}$ bacteria in the soil and reducing the rate of infection through contaminated soil reduces the value of the $\mathrm{R}_{0}$ and hence helps to contain the disease. Conversely, the parameters with negative indices are $r, d, \alpha_{p}, q, K$ and $\mu_{h}$ which means that, increasing (or decreasing) any of these parameters results to decrease (or increase) of the $\mathrm{R}_{0}$. In order to best control the disease these parameters with negative indices should be increased so as to reduce the value of the $\mathrm{R}_{0}$.

According to [4] Xcm bacterium is systemic in nature, it can invade the whole plant from the point of infection to its lateral shoots if the diseased plant is not properly removed on time. Now, leaving the diseased plant to die in the farm gives a chance of 


\section{John Joel Mapinda, Gasper Godson Mwanga and Verdiana Grace Masanja}

the $\mathrm{Xcm}$ bacteria to spread wider and hence spread of the disease. Therefore, the parameter $d$ should be carefully considered during the selection of control measures. Other parameters whose indices are more close to zero are considered to be less sensitive to the $R_{0}$, hence they can be tolerated.

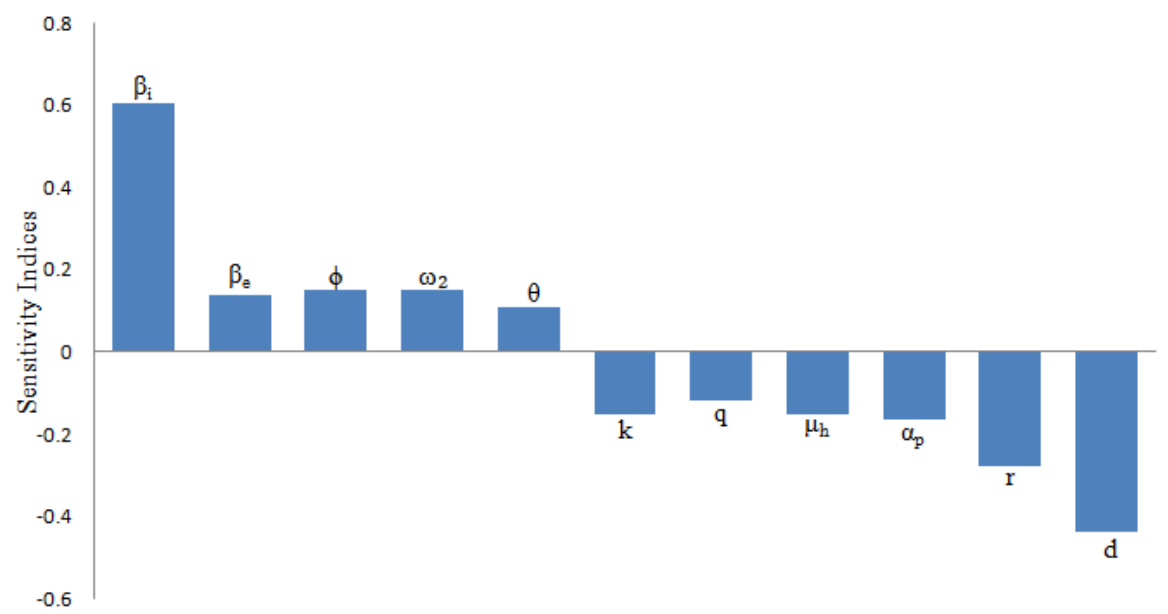

Figure 2: Sensitivity Indices for the model parameters

\subsection{Numerical simulations}

In this section we simulate the basic model to study the dynamics of BXW disease when control measures are not included. Although these results seem to be the expected behaviour, however this study has established the optimal rate that will reduce the new infections to the lowest possible level. From Figure3, it is observed that the number of susceptible plants decreases exponentially due to infection by BXW disease. The number of asymptomatic banana plants increases during the first four months of infection. After four months the number of asymptomatic plants starts to decrease while the number of symptomatic plants continues to increase. This is because most of the banana cultivars start showing symptoms after 3 months and hence reduce the number of a symptomatic plants.

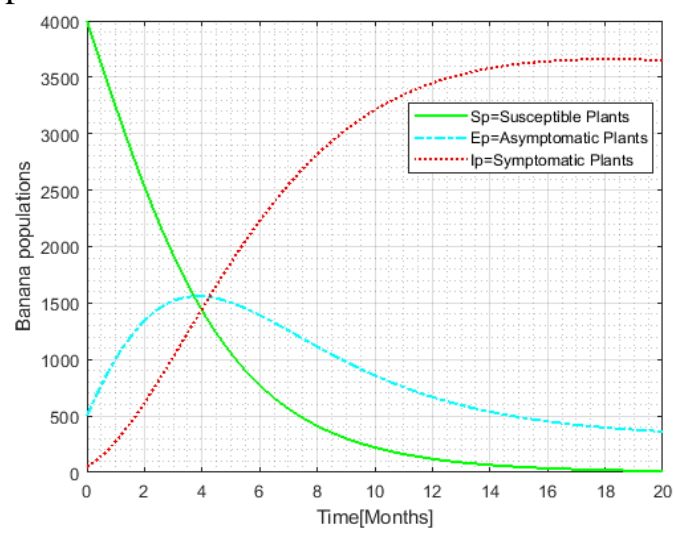

(a) Banana population

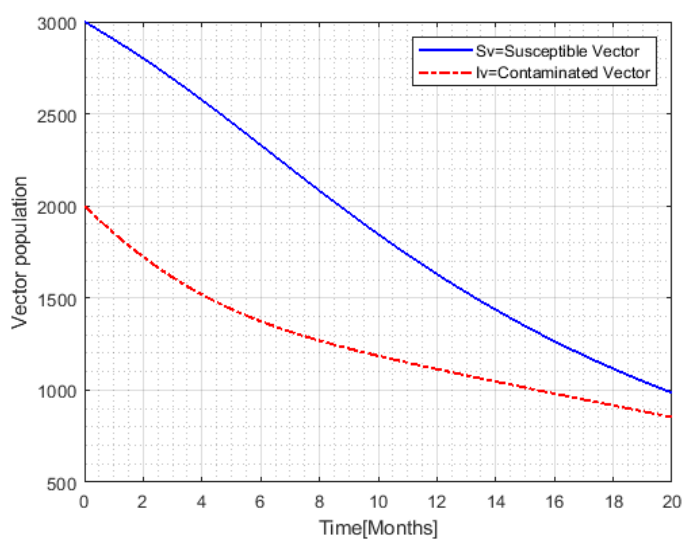

(b) Vector population

Figure 3: Population dynamics 
Modelling the Transmission Dynamics of Banana Xanthomonas Wilt Disease with Contaminated Soil

It can also be observed that without control measures, after 18 months all the banana plants will be infected with or without symptoms, which implies that there will be no susceptible plants. Figure 3 shows that the number of both susceptible and contaminated vectors in the farm decreases with time as the number of banana plants decrease. This may be attributed by low recruitment rate vs. high mortality rate.

\section{Sensitive parameters}

Figure 4 shows that as the rate of infection through farming tools contaminated with $\mathrm{Xcm}$ bacteria from symptomatic infected banana plants $\left(\beta_{i}\right)$ increases dramatically increases the number of asymptomatic and symptomatic infected banana plants. This eventually results to the increase in number of symptomatic infected plants. Figure 5 shows that increasing the rate of infection through farming tools contaminated with $\mathrm{Xcm}$ bacteria by an asymptomatic infected banana plant $\left(\beta_{e}\right)$ increases the number of secondary infections.

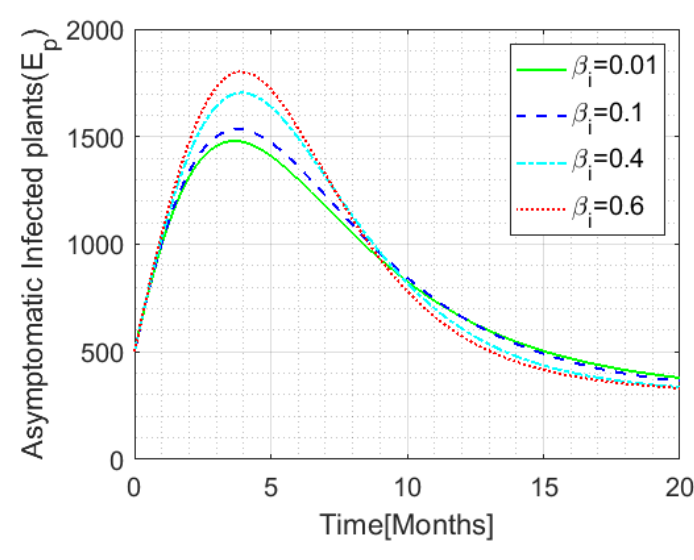

(a) Effect on $E_{p}$

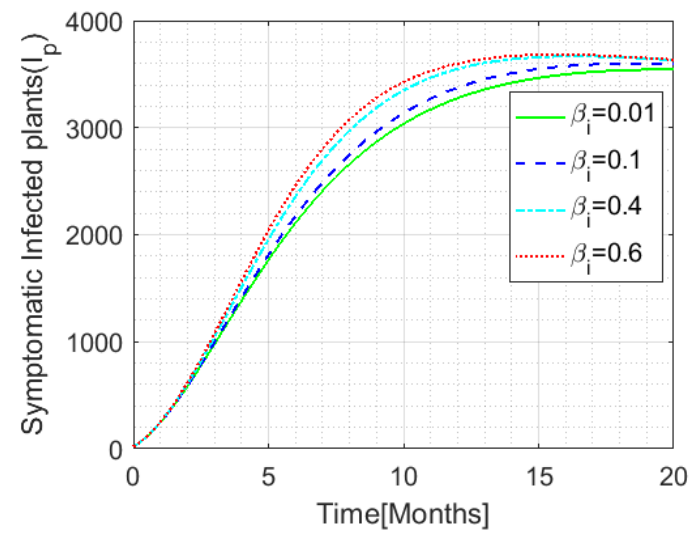

(b) Effect on $I_{p}$

Figure 4: Variations in the rate of infection through farming tools contaminated from symptomatic infected banana plants 
John Joel Mapinda, Gasper Godson Mwanga and Verdiana Grace Masanja

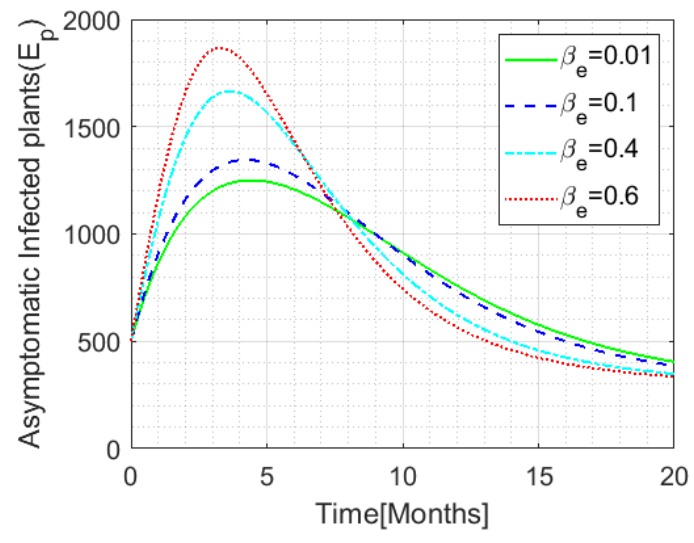

(a) Effect on $E_{p}$

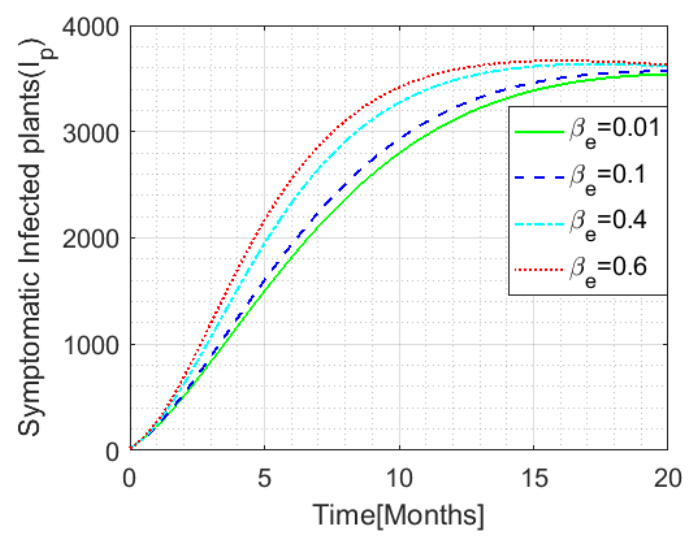

(b) Effect on $I_{p}$

Figure 5: Variations in the rate of infection through farming tools contaminated from asymptomatic infected banana plants

This implies that when performing farming activities, farmers may transmit the BXW disease unknowingly through asymptomatic infected banana plants thinking that they are health plants. In Figure6 it can be observed that increasing the rate of infection through contaminated soil $\left(\omega_{2}\right)$ increases the number of asymptomatic and symptomatic infected banana plants.

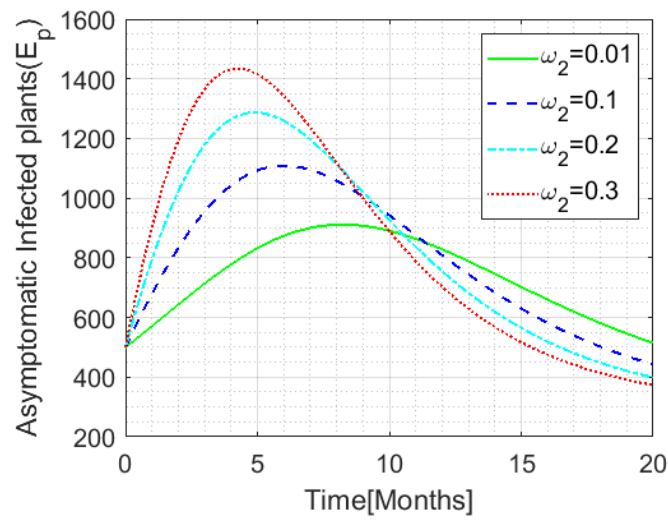

(a) Effect on $E_{p}$

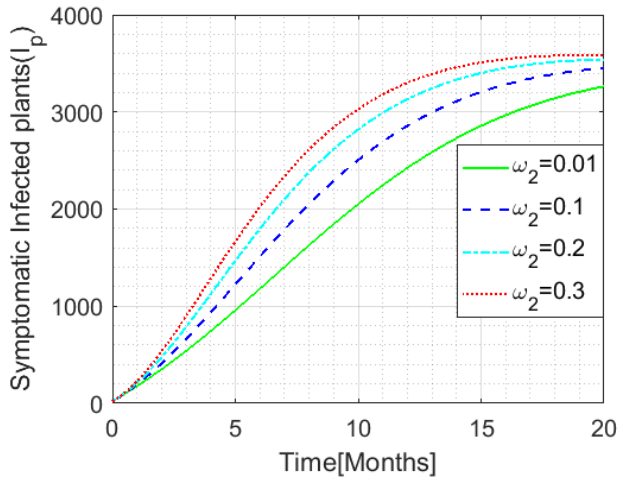

(b) Effect on $I_{p}$

Figure 6: Variations in the rate of infection through contaminated soil

With reference to Figure 7, the natural clearance rate of $\mathrm{Xcm}$ bacteria in the soil ( $\mu_{h}$ ) has an impact of on the dynamics of BXW disease. Increasing $\mu_{h}$ reduces the number of new infections generated through contaminated soil. Therefore increasing this parameter decreases the number of $\mathrm{Xcm}$ bacterium in the soil and hence reduces the number of secondary infection through contaminated soil. Furthermore, Figure 8 shows 
Modelling the Transmission Dynamics of Banana Xanthomonas Wilt Disease with Contaminated Soil

that timely removal of infected symptomatic plants reduces the number of new infections generated by a symptomatic infected banana plant.

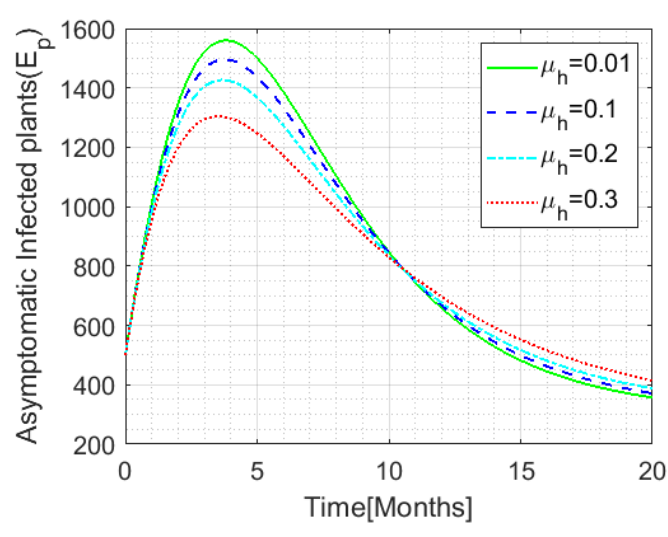

(a) Effect on $E_{p}$

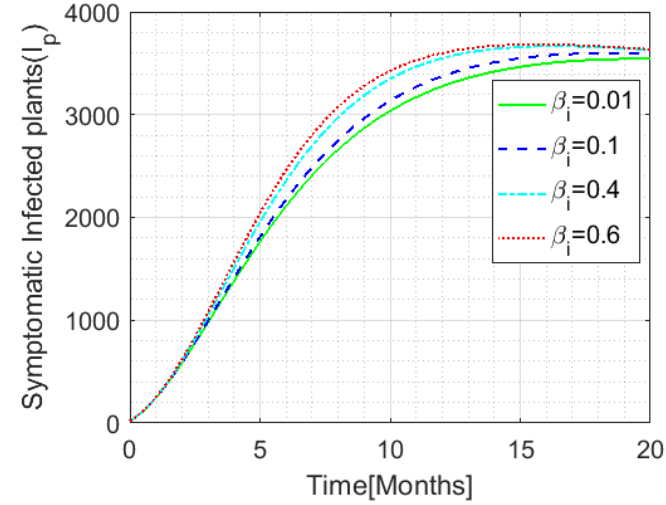

(b) mEffect on $I_{p}$

Figure 7: Variations in the natural mortality rate of $\mathrm{Xcm}$ bacteria in the soil

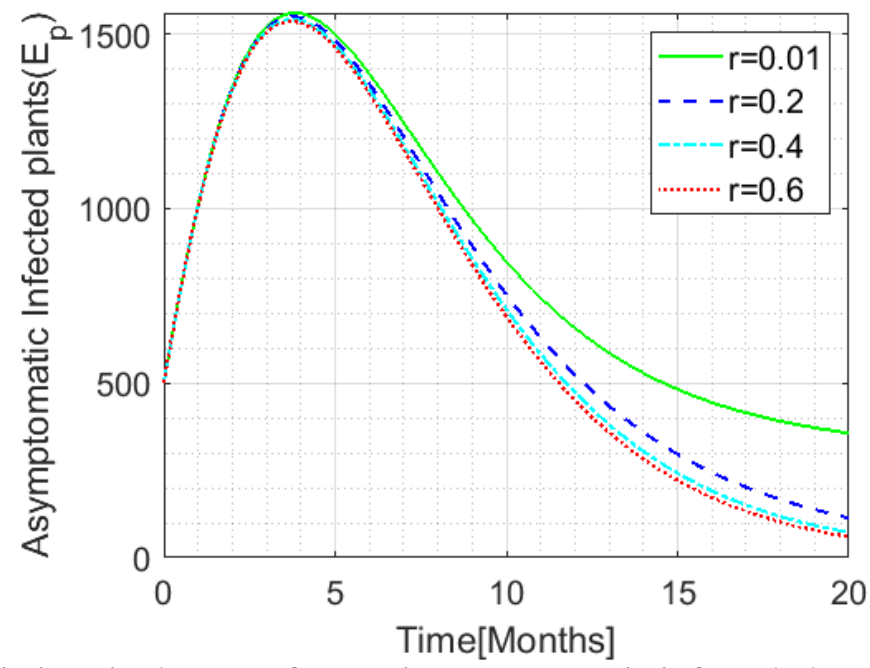

Figure 8: Variations in the rate of removing symptomatic infected plants from the farm

\section{Conclusions}

From the results, it is observed that the most sensitive parameters of the model are: the rate of infection through farming tools contaminated by Xcm bacteria from symptomatic infected plants $\left(\beta_{i}\right)$, the rate of infection through farming tools contaminated by $\mathrm{Xcm}$ bacteria from asymptomatic infected plants $\left(\beta_{e}\right)$, the rate of infection through contaminated soil $\left(\omega_{2}\right)$, rate of removing infected banana plant from the farm $(r)$, Clearance rate of bacteria in the environment $\left(\mu_{h}\right)$, vertical transmission $(\theta)$, and 
John Joel Mapinda, Gasper Godson Mwanga and Verdiana Grace Masanja

disease induced death rate of an infected banana plant $(d)$. Results from the parameter $\omega_{2}$ and $\mu_{h}$ show that contaminated soil contributes to BXW disease transmission and persistence. Thus ignoring this component of the model may lead to underestimation of $\mathrm{BXW}$ disease transmission.

Therefore, in order to best contain the disease, along with the current control measures we propose the following recommendations to scientists and technologist:

- To carry out studies that will find a way infection from infected mother plant to its suckers can be reduced or completely stopped.

- To carry out studies that will find a way to speed up the clearance rate of $\mathrm{Xcm}$ bacteria in the soil without disturbing the ecosystem so as to avoid soil inoculum which is the source of soil borne infections and persistence of the disease in farm.

- Furthermore, technology for early detection of infected plants should be brought down to the local farmers at affordable costs, this will help stakeholders to detect and remove the infected plants from the farm on time. Also farmers when performing farming activities such as harvesting, pruning, weeding and removing of the infected symptomatic banana plants should sterilize their farming tools before moving to another banana plant.

Acknowledgements. The authors thank the Nelson Mandela Institution of Science and Technology (NM-AIST) and CREATES centre of excellence for their support. Furthermore, the authors are also grateful to the reviewers for their valuable suggestions to improve the presentation of the paper.

\section{REFERENCES}

1. FAO, FAO Statistics.(2018), visited on 23 May 2019, [Online]. Available: http://fao.org/faostat/en/\#home.

2. A.Z.Geberewold, Review on impact of banana bacterial wilt (Xhantomonas campestris pv.Musacerum) in East and Central Africa, Cogent Food Agric., 5(1) (2019)1586075.

3. I.Hashim, Banana xanthomonas wilt: incidence, transmission, pathogen characterization and management options in Kagera, Mwanza and Mara regions, Sokoine University of Agriculture, 2013.

4. W.Ocimati, F.Ssekiwoko, E.Karamura, W.Tinzaara, S.Eden-Green and G.Blomme, Systemicity of $\mathrm{X}$ anthomonas campestris pv.musacearum and time to disease expression after inflorescence infection in $\mathrm{E}$ ast $\mathrm{A}$ frican highland and $\mathrm{P}$ isang $\mathrm{A}$ wak bananas in U ganda, Plant Pathol., 62(4) (2013) 777-785.

5. H.Buregyeya, J.Kubiriba, G.Tusiime, R.Kityo and F.Ssekiwoko, Role of Birds \& Bats in Long Distance Transmission of Banana Bacterial Wilt in Uganda, 2014.

6. J.Kubiriba and W.K.Tushemereirwe, Approaches for the control of banana Xanthomonas wilt in East and Central Africa, African J. Plant Sci., 8 (2014) 398404.

7. J.Nakakawa, J.Y.T.Mugisha, W.S.Michael, W.Tinzaara and E.Karamura, Banana Xanthomonas Wilt Infection: The Role of Debudding and Roguing as Control Options within a Mixed Cultivar Plantation, Int. J. Math. Math. Sci., 2017 (2017) 113.

8. J.Nakakawa, J.Y.T.Mugisha, W.S.Michael and E.Karamura, A Mathematical Model 
Modelling the Transmission Dynamics of Banana Xanthomonas Wilt Disease with Contaminated Soil

for the Dynamics of Banana Xanthomonas Wilt With Vertical Transmission and Inflorescence Infection, J. Biol. Syst., 24(1) (2016) 147-165.

9. E.H.Kweyunga, J.Tumwiine and E.Karamura, Modeling the dynamics of banana Xanthomonas wilt transmission incorporating infectious force in both asymptomatic and symptomatic stages, 2018.

10. E.H.Kweyunga, Mathematical models for the transmission and control of banana bacterial wilt epidemic, Mbarara University of Science and Technology, 2011.

11. B.Nannyonga, L.S.Luboobi, P.Tushemerirwe and M.Jabłońska-Sabuka, Using contaminated tools fuels outbreaks of Banana Xanthomonas wilt: An optimal control study within plantations using Runge-Kutta fourth-order algorithms, Int. J. Biomath., 8(5) (2015) 1550065.

12. V.Nakato, G.Mahuku and T.Coutinho, Xanthomonas campestris pv. musacearum: a major constraint to banana, plantain and enset production in central and east Africa over the past decade, Mol. Plant Pathol., 19(3) (2018)25-536.

13. C.Sivirihauma, W.Ocimati, L.Vutseme, J.Ntamwira, L.Bahati and G.Blomme, Symptomless banana suckers sourced from Xanthomonas wilt infected fields are a viable alternative for seed within infected banana-based landscapes lacking access to clean planting materials, 2017.

14. M.M.Shimwela et al., Local and regional spread of banana Xanthomonas wilt (BXW) in space and time in Kagera, Tanzania, Plant Pathol., 66(6) (2016) 10031014.

15. A.Rutikanga, G.Tusiime, G.Night, W.Ocimati and G.Blomme, Variation in nectar volume and sugar content in male flowers of Musa cultivars grown in Rwanda and their non-effect on the numbers of visiting key diurnal insect vectors of banana Xanthomonas wilt, 2016.

16. J.M.Mwebaze, G.Tusiime, W.K.Teshemereirwe and J.Kubiriba, The survival of Xanthomonas campestris pv. musacearum in soil and plant debris, African Crop Sci. J., 14(2) 2006.

17. P.den Driessche and J.Watmough, Reproduction numbers and sub-threshold endemic equilibria for compartmental models of disease transmission, Math.Biosci., 180(1-2) (2002) 29-48.

18. O.Diekmann, J.A.P.Heesterbeek and M.G.Roberts, The construction of nextgeneration matrices for compartmental epidemic models, J. R. Soc. Interface, 7(47) (2009) 873-885.

19. E.N.Lazarus, Lyapunovfunctions in epidemiological modeling, University of Namibia, 2018.

20. Z.Shuai and P.van den Driessche, Global stability of infectious disease models using Lyapunov functions, SIAM J. Appl. Math., 73(4) (2013) 1513-1532.

21. N.Chitnis, J.M.Cushing and J.M.Hyman, Bifurcation analysis of a mathematical model for malaria transmission, SIAM J. Appl. Math., 67(1) (2006) 24-45. 